\title{
COMPLICAÇÕES EM ARTROSCOPIA DE QUADRIL
}

\author{
COMPLICATIONS IN HIP ARTHROSCOPY
}

\author{
Marcos Emílio Kuschnaroff Contreras ${ }^{1}$, Rafael Barreiros Hoffmann², Lúcio Cappelli Toledo de Araújo³, \\ William Sotau Dani ${ }^{4}$, Francisco José Berral ${ }^{5}$
}

\section{RESUMO}

Objetivos: Determinar a prevalência de complicações ocorridas em uma série de casos consecutivos de artroscopia de quadril; avaliar a evolução da casuística através de uma curva de aprendizado; reconhecer as causas das complicações nas cirurgias do quadril por via artroscópica. Métodos: Foram avaliados 150 casos consecutivos submetidos à artroscopia de quadril no período de maio de 2004 a dezembro de 2008. As complicações encontradas foram classificadas sob três aspectos: sistema orgânico acometido, gravidade, grupos de 50 casos de acordo com a ordem de realização do procedimento. Utilizaram-se para a análise dos dados obtidos a estatística descritiva e o teste exato de Fisher. Resultados: Observaram-se 15 complicações (10\%) neste estudo. Dez foram complicações neurológicas, duas osteoarticulares, uma vásculo-isquêmica e duas cutâneas. Na classificação de gravidade, três foram classificadas como maiores, 12 intermediárias e nenhuma considerada menor. A incidência das complicações ao longo da curva de aprendizado não apresentou diferença estatística significativa $(p=0,16)$. Conclusões: A artroscopia de quadril é um procedimento cirúrgico de baixa morbidade, que cursa, em alguns casos, com complicações. Essas são, frequentemente, neurológicas e transitórias. Elas ocorrem, principalmente, devido à tração articular. A taxa de complicações não diminuiu com o evoluir da casuística.

Descritores - Artroscopia; Articulação do quadril; Complicações

\section{ABSTRACT}

Objectives: to determine the prevalence of complications in a series of consecutive cases of hip arthroscopy; to assess the progression of the casuistic through a learning curve; and to recognize the causes of complications in arthroscopic hip surgeries. Method: 150 consecutive cases were evaluated, who were submitted to hip arthroscopy from May 2004 through December 2008. All the surgical complications described were classified according to three aspects: organic system involved, severity, and every 50 consecutive cases. The data obtained were analyzed, and statistical analysis was carried out using Fischer's exact test. Results: We observed 15 complications (10\%) in this study; two osteoarticular, one ischemic vascular and two cutaneous. In the classification of severity, 3 were classified as very severe, 12 as intermediary and none as not severe. The incidence of complications through our learning curve showed no statistical significance $(p=0.16)$. Conclusions: Hip arthroscopy is a surgical procedure that involves low morbidity, but that still presents complications in some cases. These complications are frequently neurological and non-permanent, and are mainly due to the joint traction. The complication rate did not decrease with the progression of our casuistic.

Keywords - Arthroscopy; Hip joint; Complications

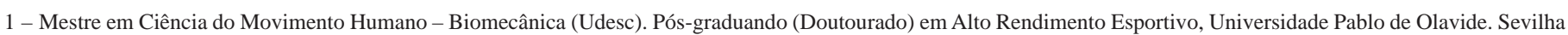
- Espanha. Chefe do Grupo de Quadril do Serviço de Ortopedia do Hospital Governador Celso Ramos - Florianópolis, SC.

2 - Médico Residente do Serviço de Ortopedia do Hospital Governador Celso Ramos -Florianópolis, SC.

3 - Médico Ortopedista da Clínica Catarinense do Quadril - Florianópolis, SC.

4 - Médico Ortopedista - Clinitrauma - Lages, SC.

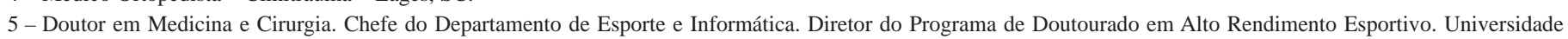
Pablo de Olavide. Sevilha - Espanha
} 


\section{INTRODUÇÃO}

Artroscopia é um método cirúrgico que permite a abordagem de diversas articulações de maneira pouca invasiva, permitindo, dessa forma, diminuição da morbidade ocasionada pelos grandes procedimentos, alta hospitalar precoce e período de reabilitação menor. Atualmente, a cirurgia artroscópica é o procedimento cirúrgico ortopédico mais comumente realizado nos EUA $^{(1)}$. A artroscopia de quadril é indicada como método diagnóstico e terapêutico e suas indicações incluem lesão labral, remoção de corpos livres intra-articulares, impacto fêmoro-acetabular, entre outros ${ }^{(2-5)}$.

As revisões descritas na literatura citam taxas variáveis de complicações. Alguns autores citam que elas variam entre 0,5 e $5 \%^{(2)}$, enquanto outros mencionam de 0,5 a $6,4 \%{ }^{(3)}$. A maior parte dos trabalhos atribui as complicações, principalmente, à tração $\operatorname{articular}^{(4,5)}$.

O objetivo deste trabalho é determinar a prevalência de complicações nos diversos procedimentos artroscópicos realizados através de artroscopia de quadril por meio de um estudo retrospectivo do tipo série de casos, sendo eles consecutivos, avaliar a evolução de nossa casuística através de uma curva de aprendizado e reconhecer as causas dessas complicações.

\section{MÉTODOS}

Trata-se de um estudo retrospectivo tipo série de casos consecutivos com 150 pacientes, os quais foram submetidos à cirurgia de quadril por via artroscópica, por qualquer que seja a indicação, no período de maio de 2004 a dezembro de 2008. Desses, 69 (46\%) eram do sexo masculino e 81 (54\%) do feminino. A idade média dos pacientes foi de 37,25 anos (12 a 58 anos). No que diz respeito ao lado do quadril submetido ao procedimento cirúrgico, o direito prevaleceu em 84 (56\%) casos, sendo o esquerdo submetido em 63 (42\%) pacientes; três (2\%) pacientes foram submetidos à artroscopia de quadril bilateral.

Todos os pacientes foram avaliados no pré e pósoperatórios pelo mesmo observador, submetidos a tratamento cirúrgico por ele.

Em todos os nossos pacientes utilizamos anestesia geral. A posição utilizada em nossa casuística foi o decúbito dorsal, onde o poste perineal foi posicionado contra a região medial da coxa do quadril submetido ao procedimento cirúrgico, a fim de gerar um vetor de força com sentido lateral. O quadril do lado cirúr- gico foi colocado em rotação interna de $20^{\circ}$, flexão de $10^{\circ}$ e força em adução era realizada. O membro contralateral foi posicionado e tracionado o suficiente para que houvesse equilíbrio do paciente na mesa de cirurgia.

Após o posicionamento do paciente, o membro a ser submetido ao procedimento cirúrgico foi tracionado visando obter aumento do espaço intra-articular até que o sinal do vácuo estivesse aparente à radioscopia. Após tais procedimentos, iniciou-se a distensão da cápsula articular através de injeção de salina, cerca de 10 a 20ml, por punção com agulha. Utilizamos em todo o procedimento uma bomba de infusão de solução salina com pressão inicial de 60mmHg em fluxo máximo, para manter a distensão do espaço intra-articular.

O procedimento artroscópico do quadril comumente utilizado por nossa equipe envolveu quatro portais de acesso, descritos por Byrd ${ }^{(6)}$ : anterior, anterolateral, posterolateral e intermediário. Na maioria dos casos utilizamos apenas dois portais (anterior e anterolateral).

Os dados obtidos sobre complicações em artroscopia de quadril foram analisados sob três aspectos: tipo de complicação (neurológico, osteoarticular, vásculo-isquêmicas e cutâneas); gravidade, tal qual Souza et $a l^{(7)}$, que subdividiram as complicações em três categorias de gravidade: maiores, complicações que foram definitivas ou necessitaram de tratamento cirúrgico para sua correção; intermediárias, aquelas transitórias com recuperação completa após tratamento clínico; e, por fim, menores, quando foram resolvidas no ato cirúrgico; e incidência de complicações na evolução de nossa casuística em uma curva de aprendizado, subdividindo os casos em grupos de 50 pacientes, por ordem de ocorrência do procedimento cirúrgico.

Utilizamos a estatística descritiva e o teste exato de Fisher para análise dos dados, consideramos resultados com significância estatística aqueles em que p <0,05.

Este trabalho foi aprovado pelo Comitê de Ética em Pesquisa do Hospital Governador Celso Ramos em reunião no dia 12 de março de 2009, sob o n. ${ }^{\circ}$ 2008/0038.

\section{RESULTADOS}

Observamos um total de 15 (10\%) complicações em nossa série (Tabela 1). Dessas, 10 foram neurológicas, duas osteoarticulares, uma vásculo-isquêmica e duas cutâneas. Ao agruparem-se tais complicações no quesito gravidade, três apresentaram-se como maiores, 12 como intermediárias e nenhuma foi considerada menor. 
Tabela 1 - Lista de pacientes, suas complicações e demais dados coletados

\begin{tabular}{c|c|c|c|c|c|c}
\hline Caso & Sexo & Idade & Lado & Complicação & Tipo & Gravidade \\
\hline 9 & F & 41 & E & Neuropraxia do pudendo & Neurológica & Intermediária \\
\hline 14 & F & 35 & D & Edema de vulva & Vásculo-isquêmica & Intermediária \\
\hline 35 & F & 46 & D & Deiscência de sutura & Cutânea & Intermediária \\
\hline 36 & F & 36 & D & Neuropraxia do pudendo & Neurológica & Intermediária \\
\hline 57 & M & 30 & D & Neuropraxia do pudendo & Neurológica & Intermediária \\
\hline 79 & F & 50 & D & Instabilidade & Osteoarticular & Grave \\
\hline 80 & F & 24 & E & Neuropraxia do ciático & Neurológica & Intermediária \\
\hline 81 & F & 26 & D & Neuropraxia do pudendo & Neurológica & Intermediária \\
\hline 87 & F & 35 & E & Parestesia permanente & Neurológica & Grave \\
\hline 92 & M & 24 & E & Neuropraxia do ciático & Neurológica & Intermediária \\
\hline 98 & F & 49 & D & Instabilidade & Osteoarticular & Grave \\
\hline 107 & F & 26 & E & Neuropraxia do ciático & Neurológica & Intermediária \\
\hline 112 & F & 31 & E & Neuropraxia do ciático & Neurológica & Intermediária \\
\hline 122 & F & 31 & E & Neuropraxia do ciático & Neurológica & Intermediária \\
\hline 123 & F & 25 & D & Deiscência de sutura & Cutânea & Intermediária \\
\hline
\end{tabular}

No grupo de complicações maiores tivemos dois casos de quadris displásicos que evoluíram para instabilidade e foram submetidos à artroplastia total de quadril (complicação osteoarticular); outro caso apresentou-se com parestesia permanente de membros inferiores, sem apresentar melhora após dois anos de seguimento (complicação neurológica). Entre as complicações intermediárias, as mais comuns foram neurológicas, sendo as neuropraxias perineais, oito casos (5,33\% de nossa casuística), as mais frequentes. Ainda com relação às complicações intermediárias neurológicas, um paciente $(0,6 \%$ de nossa série) apresentou neuropraxia do nervo ciático, com melhora motora após três dias e melhora sensitiva após quatro meses. Houve um caso $(0,6 \%$ dos nossos pacientes) de complicação vásculo-isquêmica entre as intermediárias - edema transitório de vulva. O grupo de complicações de gravidade intermediária apresentou duas (1,33\% dos nossos casos) complicações cutâneas, as quais foram de deiscência de sutura e apresentaram melhora com tratamento clínico (Figura 1).

Não observamos nenhum caso de trombose venosa profunda, infecção profunda, lesão labral, condral ou da cápsula articular bem como quebra de instrumental cirúrgico.

Com relação aos sistemas orgânicos acometidos em cada um dos grupos, observamos que o grupo 1 apresentou duas complicações neurológicas, uma complicação vásculo-isquêmica e uma cutânea. Já no grupo 2,

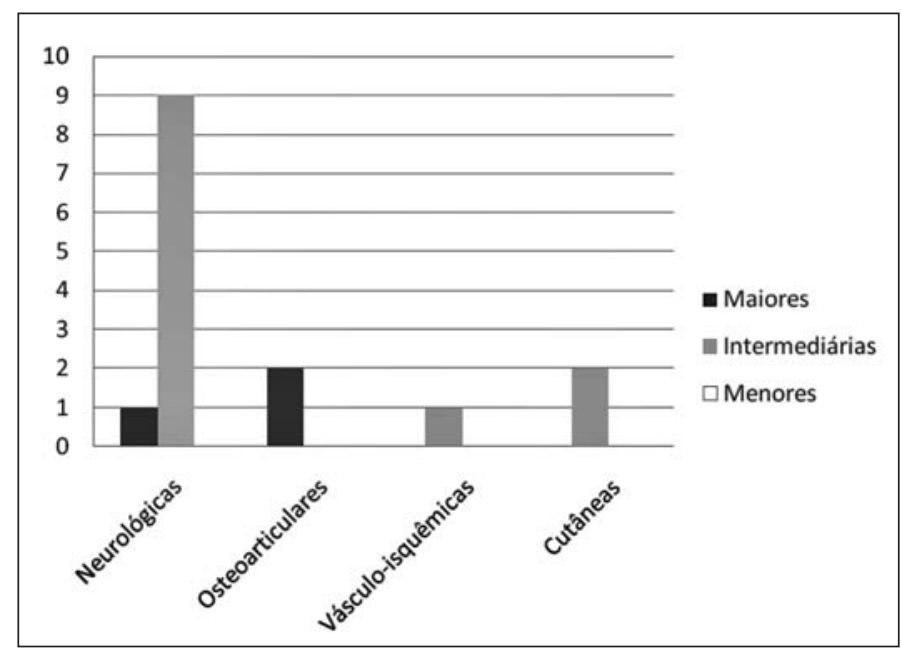

Figura 1 - Frequência das complicações separadas por sistema orgânico acometido e por sua gravidade

observamos cinco complicações neurológicas e duas osteoarticulares. E, no grupo 3, tivemos três complicações neurológicas e uma cutânea (Figura 2)

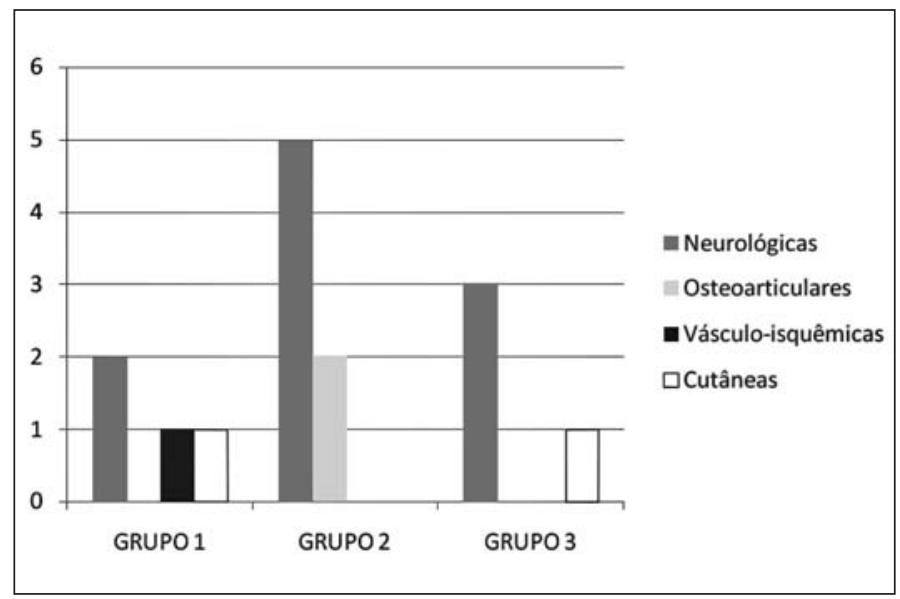

Figura 2 - Frequência das complicações conforme o Sistema Orgânico acometido durante a curva de aprendizado

\section{ANÁLISE ESTATÍSTICA}

Dividimos nossa série em três grupos de 50 pacientes, em relação à ordem de realização do procedimento cirúrgico. Tivemos quatro complicações intermediárias em cada um dos grupos e três complicações maiores no grupo 2. Não encontramos diferença estatística significante entre o número de complicações do grupo 1 e do grupo 2 $(\mathrm{p}=0,16)$ tampouco entre o grupo 2 e grupo $3(\mathrm{p}=0,16)$. Assim, não houve diferença estatística significante do número de complicações durante a nossa curva de aprendizado, apesar de o grupo 2 ter um número absoluto de complicações (sete) maior do que o dos outros (Figura 3). 


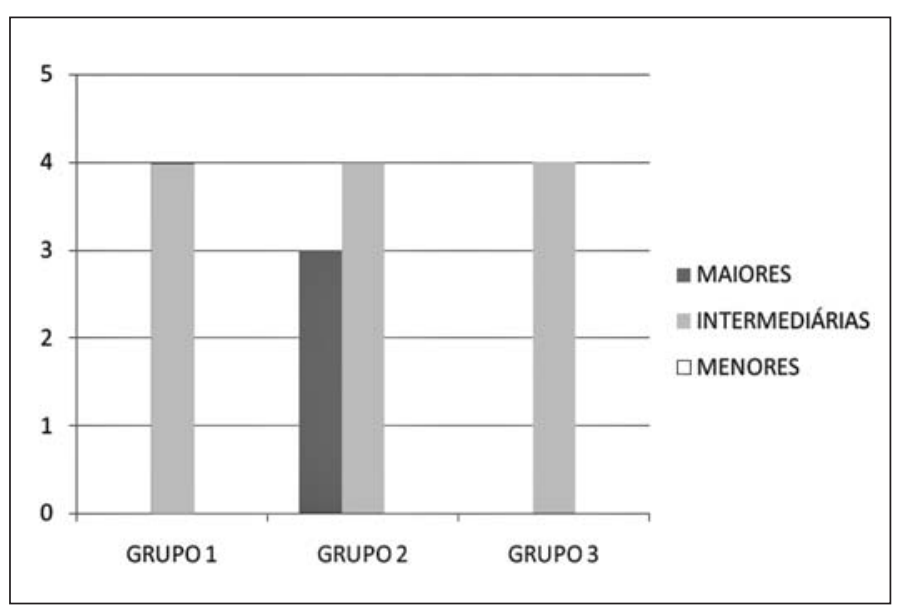

Figura 3 - Frequência das complicações conforme a curva de aprendizado

\section{DISCUSSÃO}

Em revisão da literatura, McCarthy e Lee ${ }^{(2)}$ citaram uma taxa de 0,5 a $5 \%$ de complicações em procedimentos artroscópicos do quadril, enquanto Smart et $a l^{(3)}$ apresentaram taxa de 0,5 a $6,4 \%$, sendo que ambos os autores afirmaram serem as neuropraxias as complicações mais comuns. As complicações ocorridas na artroscopia de quadril são relacionadas, principalmente, com a tração articular e realização de portais ${ }^{(4)}$. Rodeo et $a l^{(5)}$ mostrou que lesões neurológicas ocorrem por lesão direta devido à localização incorreta dos portais, excessiva força de tração ou por compressão do poste perineal.

Percebemos, em nossa série, 11 complicações (73,33\% do nosso total) atribuíveis à tração. Um dos casos foi considerado grave devido à persistência da parestesia de membros inferiores durante todo o período de seguimento (dois anos), mesmo o paciente tendo eletroneuromiografia normal. Kim et $a l^{(8)}$ relataram um caso de distrofia simpático-reflexa após artroscopia do quadril que não apresentou resolução após dois anos de seguimento.

Outros nove pacientes apresentaram neuropraxias transitórias e foram considerados intermediários na classificação de gravidade, devido à melhora com tratamento clínico. Funke e Munzinger ${ }^{(9)}$ observaram um caso de neuropraxia transitória; Sampson ${ }^{(10)}$, em um estudo com 1.001 pacientes, apresentou 20 casos de neuropraxias transitórias; Griffin e Villar(11), em análise de 640 casos consecutivos, apresentaram quatro episódios de neuropraxias transitórias; Clarke et $\mathrm{al}^{(4)}$, em 1.054 casos consecutivos, relataram quatro casos de neuropraxias; Souza et $a^{(7)}$ apresentaram cinco casos de neuropraxias transitórias; Byrd e Jones ${ }^{(12)}$ observaram um caso de neuropraxia transitória em estudo realizado com 38 procedimentos artroscópicos em uma amostra de 35 pacientes; Kim et $a l^{(8)}$ relataram quatro casos que apresentaram neuropraxias transitórias. Dienst et al $^{(13)}$, em série de artroscopias de quadril no compartimento periférico sem uso de tração, apresentaram um caso de neuropraxia transitória. É importante salientar que McCarthy e Lee ${ }^{(2)}$, em sua revisão da literatura, alertaram que as principais medidas preventivas para evitar lesões neurológicas são o posicionamento correto do paciente e a tração adequada.

Outro caso de complicação relacionada com a tração que observamos foi edema de vulva - única complicação vásculo-isquêmica, a qual foi considerada de gravidade intermediária. Tal intercorrência aconteceu quando nos faltou a espuma de proteção perineal de uso rotineiro e utilizamos uma proteção perineal com outro tipo de espuma. Funke e Munzinger ${ }^{(9)}$ relataram um caso de hematoma de grandes lábios; Clarke et $\mathrm{al}^{(4)}$ observaram um caso de lesão vaginal; Griffin e Villar ${ }^{(11)}$ apresentaram um caso de lesão em região de vagina; Souza et $a l^{(7)}$ descreveram um episódio de edema de vulva, o qual consideraram complicação intermediária, e um caso de necrose parcial da pele do escroto, considerada complicação grave, que foi corrigida através de cirurgia plástica.

Em nossa série, percebemos dois casos (13,33\% das complicações) relacionados com os portais cirúrgicos que foram classificados como cutâneos. Ambos apresentaram-se na forma de deiscência de sutura e classificamos como intermediários devido à evolução benigna após o tratamento clínico. Não encontramos relatos de infecção superficial na literatura. Tal fato pode ter ocorrido em virtude de que essa complicação não pode ser atribuída somente à artroscopia de quadril, mas sim aos procedimentos cirúrgicos em geral. Diversos autores relatam outras intercorrências relacionadas com os portais. Clarke et $a^{(4)}$ observaram dois casos de sangramento e dois casos de hematoma nos portais; Griffin e Villar ${ }^{(11)}$ relataram um caso de sangramento no portal e um caso de hematoma na ferida operatória.

Não tivemos, em nosso estudo, casos de infecção profunda. Clarke et Il $^{(4)}$ relataram um caso de artrite séptica, sendo Staphylococcus aureus sensível à clindamicina o agente etiológico, após 26 dias da realização de artroscopia de quadril para tratamento de osteocondromatose. 
Observamos duas complicações (13,33\% desses casos) osteoarticulares no nosso estudo. Ambas aconteceram em pacientes que foram submetidos ao procedimento artroscópico devido à displasia de quadril. Os dois pacientes evoluíram com instabilidade e foram submetidos à artroplastia total de quadril para correção do problema. Acreditamos que ambos os casos não tiveram indicação adequada para artroscopia. Alguns podem considerar isso como mau resultado, e não como complicação; por esse motivo, pode não estar relacionada em outras series.

Com relação às complicações osteoarticulares, Sampson ${ }^{(10)}$ relatou três casos de lesão iatrogênica da cartilagem articular, atribuídos à tração inadequada; um caso de necrose avascular da cabeça do fêmur, em decorrência de um distúrbio da artéria circunflexa femoral medial; um caso de fratura do colo femoral, devido à grande ressecção do colo femoral; e, mais tardiamente, um caso de ossificação heterotópica. Sussmann et $a l^{(14)}$, em estudo com cadáveres, mostraram que a artéria circunflexa femoral medial é protegida pelo trocanter maior quando o portal posterolateral é utilizado; Griffin e Villar ${ }^{(11)}$ apresentaram um caso de bursite trocantérica, a qual respondeu ao tratamento com injeção de corticoides. Clarke et al $^{(4)}$ relataram, também, um caso de bursite trocantérica que apresentou resolução após o uso de terapia com corticoide local. Souza et al $^{(7)}$ relataram duas complicações relativas ao aparelho osteoarticular, sendo um paciente submetido à artroscopia de quadril devido a impacto fêmoro-acetabular, o qual evoluiu com instabilidade do quadril, necessitando de artroplastia total de quadril para resolução da intercorrência - considerada complicação de gravidade maior em seu trabalho -, e em outro paciente, submetido ao procedimento artroscópico devido a impacto fêmoro-acetabular, aconteceu fratura por estresse do colo femoral, sem desvio, que foi tratada conservadoramente e evoluiu com consolidação após oito semanas - classificada como complicação intermediária. Dienst et $\mathrm{al}^{(13)}$, em seu estudo sobre artroscopia de quadril no compartimento periférico sem uso de tração, apresentaram três lesões da cartilagem articular, uma liberação de osteófito - com resolução no ato - e dez lesões parciais de cápsula articular.

Não observamos em nossa série casos de extravasamento de fluido para o espaço retroperitoneal ou abdominal. Sampson ${ }^{(10)}$ observou dez casos de extravasamento de fluido para a cavidade abdominal; Barlett et $a l^{(15)}$ relataram um caso de extravasamento de fluido, em grande quantidade, para cavidade abdominal, atribuído à fratura de acetábulo, que resultou em parada cardiorrespiratória, onde somente se conseguiu estabilizar hemodinamicamente o paciente após extração de parte do líquido da cavidade.

Não reconhecemos, em nossa casuística, casos de trombose venosa profunda. Souza et $a l^{(7)}$ observaram um caso de trombose venosa profunda que apresentou recuperação completa, sem sequelas, após tratamento clínico. McCarthy e Lee ${ }^{(2)}$ relataram um caso de trombose venosa profunda após um mês de pós-operatório em paciente com deficiência de fator $\mathrm{V}$ de Leiden. Bushnell et al ${ }^{(16)}$, em revisão com mais de 5.500 casos, não encontraram casos de trombose venosa profunda, tampouco recomendações específicas para profilaxia desse evento.

Não observamos casos de quebras de instrumental cirúrgico em nossa série. Sampson ${ }^{(10)}$ relatou três quebras de instrumental; enquanto Griffin e Villar ${ }^{(11)}$ referiram dois casos de quebras de instrumental; Clarke et $a l^{(4)}$ apresentaram dois casos de quebra de instrumental; Souza et al $^{(7)}$ observaram dois casos de quebra de material cirúrgico, que consideraram complicações de gravidade menor em virtude da resolução durante o transoperatório.

Ao contrário do que Sampson ${ }^{(10)}$ relatou, e corroborando o que Souza et $a l^{(7)}$ observaram, não reconhecemos diminuição das complicações com o decorrer de nossa casuística. Tal fato pode ser atribuído ao aumento da complexidade dos procedimentos artroscópicos realizados por nossa equipe, apesar da evolução das técnicas em artroscopia do quadril e dos equipamentos utilizados para o procedimento cirúrgico. Apesar de não existir diferença estatística significante no numero de complicações durante a nossa curva de aprendizado, o período entre os casos 51 e 100 teve o maior numero de complicações absolutas (sete) e dessas, três foram complicações maiores. Foi nesse período, aparentemente, que o cirurgião se tornou mais ousado, podendo ter indicado inadequadamente a técnica para alguns casos, tentando dar passos mais largos para busca de seu aperfeiçoamento.

Além disso, poucas são as séries que incluem os primeiros casos de cada um dos autores. Encontramos, em nossa revisão, apenas três artigos que se enquadram nesse quesito: Sampson ${ }^{(10)}$, Clarke et $a l^{(4)}$ e Souza et $a l^{(7)}$. Nas demais series, não há referências sobre a inclusão ou não dos casos iniciais. 
A artroscopia do quadril é uma nova ferramenta cirúrgica que se mostrou segura e eficaz. Com a correta indicação, pacientes bem selecionados e maior experiência do cirurgião, a tendência é diminuir ainda mais as complicações, tornando o método a melhor opção para tratamento das patologias intra-articulares do quadril.

\section{CONCLUSÕES}

A artroscopia de quadril é um procedimento cirúrgico que permite a abordagem de tal articulação de maneira pouco invasiva, mas que cursa com algumas complicações. Tais complicações, frequentemente, neurológicas e transitórias. Elas ocorrem, principalmente, devido à tração articular realizada visando o aumento do espaço intra-articular.

Nossa taxa de complicações não apresentou diminuição com o evoluir de nossa casuística e está de acordo com o encontrado na literatura.

\section{REFERÊNCIAS}

1. Villar RN. Arthroscopy. BMJ. 1994;308(6920):51-3.

2. McCarthy JC, Lee JA. Hip arthroscopy: indications, outcomes, and complications. Bone Joint Surg Am.2005;87:1138-45.

3. Smart LR, Oetgen M, Noonan B, Medvecky M. Beginning hip arthroscopy: indications, positioning, portals, basic techniques, and complications. Arthroscopy. 2007;23(12):1348-53.

4. Clarke MT, Arora A, Villar RN. Hip arthroscopy: complications in 1054 cases. Clin Orthop Relat Res. 2003;(406):84-8.

5. Rodeo SA, Forster RA, Weiland AJ. Neurological complications due to arthroscopy. J Bone Joint Surg Am. 1993;75(6):917-26.

6. Byrd JW. Operative hip arthroscopy. 2nd ed. New York: Springer; 2004.

7. Souza BGS, Polesello G, Honda E, Ono NK, Guimaraes RP, Ricioli W. Complications in hip arthroscopy. In: Annual Meeting Podium Presentations; 2009, Fevereiro 25-27; Las Vegas, EUA: American Association of Orthopaedic Surgeons.

8. Kim SJ, Choi NH, Kim HJ. Operative hip arthroscopy. Clin Orthop Relat Res. 1998;(353):156-65.

9. Funke EL, Munzinger U. Complications in hip arthroscopy. Arthroscopy. $1996 ; 12(2): 156-9$

10. Sampson TG. Complications of hip arthroscopy. Tech Orthop. 2005;20(1):63-6.

11. Griffin DR, Villar RN. Complications of arthroscopy of the hip. J Bone Joint Surg Br. 1999;81(4):604-6.

12. Byrd JW, Jones KS. Prospective analysis of hip arthroscopy with 2-year followup. Arthroscopy. 2000;16(6):578-87.

13. Dienst M, Gödde S, Seil R, Hammer D, Kohn D. Hip arthroscopy without traction: in vivo anatomy of the peripheral hip joint cavity. Arthroscopy. 2001; 17(9):924-31

14. Sussmann PS, Zumstein M, Hahn F, Dora C. The risk of vascular injury to the femoral head when using the posterolateral arthroscopy portal: cadaveric investigation. Arthroscopy. 2007;23(10):1112-5.

15. Barlett CS, DiFelice GS, Buly RL, Quinn TJ, Green DST, Helfet DL. Cardiac arrest as a result of intraabdominal extravasation of fluid during arthroscopy removal of a loose body from the hip joint of a patient with an acetabular fracture. J Orthop Trauma. 1998;12(4):294-9.

16. Bushnell BD, Anz AW, Bert JM. Venous thromboembolism in lower extremity arthroscopy. Arthroscopy. 2008;24(5):604-11. 